\title{
When can attention influence binocular rivalry?
}

\author{
Kevin C. Dieter ${ }^{1,2} \cdot$ Michael D. Melnick ${ }^{2}$ - Duje Tadin ${ }^{2,3}$
}

Published online: 22 April 2015

(C) The Psychonomic Society, Inc. 2015

\begin{abstract}
Attentional influence over perception is particularly pronounced when sensory stimulation is ambiguous, where attention can reduce stimulus uncertainty and promote a stable interpretation of the world. However, binocular rivalry, an extensively studied visual ambiguity, has proved to be comparatively resistant to attentional modulation. We hypothesize that this apparent inconsistency reflects fluctuations in the degree of unresolved competition during binocular rivalry. Namely, attentional influence over rivalry dynamics should be limited to phases of relatively unresolved stimulus competition, such as ends of individual dominance periods. We found that transient, feature-based cues congruent with the dominant stimulus prolonged dominance durations, while cues matching the suppressed stimulus hastened its return to dominance. Notably, the effect of cues depended on when the cues are presented. Cues presented late, but not early, during a given episode of perceptual dominance influenced rivalry dynamics. This temporal pattern mirrors known changes in the relative competitive dynamics of rival stimuli, revealing that selective effects occur only during temporal windows containing weak resolution of visual competition. In conclusion,
\end{abstract}

Kevin C. Dieter

kevin.dieter@vanderbilt.edu

1 Vanderbilt Vision Research Center and Department of Psychology, Vanderbilt University, 301 Wilson Hall, 111 21st Avenue South, Nashville, TN 37240, USA

2 Department of Brain and Cognitive Sciences and Center for Visual Science, University of Rochester, Rochester, NY 14627, USA

3 Department of Ophthalmology, University of Rochester School of Medicine, Rochester, NY 14642, USA these findings reveal that unresolved competition, which gates attention across a variety of domains, is also crucial in determining the susceptibility of binocular rivalry to selective influences.

Keywords Attention: Selective · Binocular vision . Rivalry/Bistable Perception · Visual context

\section{Introduction}

As our visual system attempts to construct a meaningful representation of the external world, it frequently encounters input with multiple viable interpretations. In these ambiguous cases, other sources of information such as context and attention typically have a significant influence on perception - in fact, the influences of such factors on perception are perhaps greatest, and their effects most useful, in cases where incoming visual information is most uncertain. This has made visual bistability a useful domain within which to investigate effects of context and attention on visual processing. For example, when viewing the bistable Necker cube (Necker, 1832), unambiguous contextual information can strongly bias perception in favor of the surrounding context (Sundareswara \& Schrater, 2008). Voluntary attention can also bias perception in favor of the attended cube perspective (Meng \& Tong, 2004; Toppino, 2003). Similar modulations are seen for other forms of visual bistability including apparent motion (Suzuki \& Peterson, 2000) and structure-from-motion (Hol, Koene, \& van Ee, 2003).

Interestingly, binocular rivalry, a broadly studied form of visual bistability that occurs when incompatible images are presented to the two eyes, does not fit this pattern of attentional susceptibility. Observers are largely unable to 
selectively modulate fluctuations between rival stimuliexcept under certain conditions, such as at the onset of rivalry (Chong \& Blake, 2006; Mitchell, Stoner, \& Reynolds, 2004; Ooi \& He, 1999), or while performing a demanding perceptual task during rivalry (Chong, Tadin, \& Blake, 2005; Hancock \& Andrews, 2007; Helmholtz, 1925). Although the presence of stimulus competition is thought to drive visual attention (Desimone \& Duncan, 1995), the stimulus conflict that persists throughout binocular rivalry is hard to bias with attention. For example, if observers are instructed to try to "hold" one of two rival percepts dominant, they are unable to do so (Blake, 1988; Meng \& Tong, 2004). This differentiates binocular rivalry from other forms of visual bistability, suggesting a difference in the competitive interactions underlying binocular rivalry. Specifically, the low-level nature of interocular interactions that occur during binocular rivalry (Blake, 1989; Stuit, Paffen, Van Der Smagt, \& Verstraten, 2014) likely limit its attentional susceptibility (Dieter \& Tadin, 2011).

The strong influence of attention at the initial onset of binocular rivalry (Chong \& Blake, 2006; Mitchell et al., 2004; Ooi \& He, 1999) demonstrates that the conditions necessary for selective modulation are at least temporarily present. A key aspect of this initial period of rival interactions is that it lacks a complete resolution of rivalry conflict; for the first $150 \mathrm{~ms}$, both rival stimuli are visible (Wolfe, 1983). After this initial phase of unresolved competition, binocular rivalry consists of extended periods of relatively stable resolution (i.e., when a single image is perceptually dominant). These later periods are associated with weakened effects of attention (Mitchell et al., 2004). However, recent results suggest that even while one of two images remains perceptually dominant, the underlying representations of the images are in fact creeping closer together; at a percept's onset, probe detection performance is best in the dominant eye and poorest in the suppressed eye, but this performance difference gradually disappears as the percept extends in time (Alais, Cass, O'shea, \& Blake, 2010). In other words, as a percept nears the end of its dominance period, it weakens, while the suppressed stimulus gradually becomes stronger - conditions indicating decreased resolution of underlying stimulus competition.

The presence of unresolved visual competition is associated with strong effects of visual attention (Beck \& Kastner, 2009; Desimone \& Duncan, 1995). Therefore, we expect a greater influence of attention near the end of individual dominance periods (Dieter \& Tadin, 2011) in accordance with the reduction in resolved conflict between the two images (Alais et al., 2010).

We tested this hypothesis by presenting transient, task-irrelevant, exogenous cues with various stimulus onset asynchronies (SOAs) relative to the start of perceptual dominance epochs (Fig. 1) and examined their effect on each percept's duration. We found that cues congruent with the currently dominant stimulus prolonged its dominance duration, while cues matching the suppressed stimulus accelerated its return to dominance (Experiment 1). Importantly, these effects occurred with a great deal of temporal precision - effects were seen only on percepts that, if not for the presentation of the cue, would have ended within about $1 \mathrm{~s}$. Evidently, only the tail end of a percept's period of dominance is susceptible to selective influences during binocular rivalry. When considered along with results from studies showing attentional control over initial onsets of binocular rivalry (Chong \& Blake, 2006; Mitchell et al., 2004; Ooi \& He, 1999), a period before clear resolution of rivalry conflict (Wolfe, 1983), our finding suggests a key role of unresolved competition in driving selective control of perception during binocular rivalry. We also demonstrate that our effects are not driven by spatial interactions between cues and rival stimuli (Experiment 2), suggesting the parsimonious explanation that our results reflect exogenous effects of feature-based attention.

\section{Method}

\section{Observers}

Ten observers (four female) participated in Experiment 1, and seven observers (three female; four had previously completed Experiment 1) participated in Experiment 2. Two additional observers (one author: KD) were recruited for a follow-up experiment conducted at Vanderbilt University (collinearity control condition in Experiment 2, Fig. 4c). With respect to sample sizes, our initial goal was to test ten observers in the main experiment (Experiment 1) and fewer subjects in other experiments. Our sample sizes compare favorably to previous studies on this topic (e.g., frequently cited Chong, Tadin, \& Blake, 2005 tested only four observers). Importantly, effects of attention were found for all ten observers individually in the main experiment (Fig. 2b) and the critical time-course analysis (Fig. 3b) was conducted only after data collection ended. All observers were naïve as to the purpose of the experiments (except author KD in the collinearity control condition) and gave informed consent prior to participation in accordance with protocols approved by the Institutional Review Boards at University of Rochester and Vanderbilt University. All observers had normal or corrected-to-normal vision.

\section{Apparatus}

Experimental stimuli were generated using MATLAB and the Psychophysics Toolbox (Brainard, 1997; Pelli, 1997). All stimuli at both institutions were presented on linearized Sony GDM-FW900 CRT monitors. Experiments conducted 
a
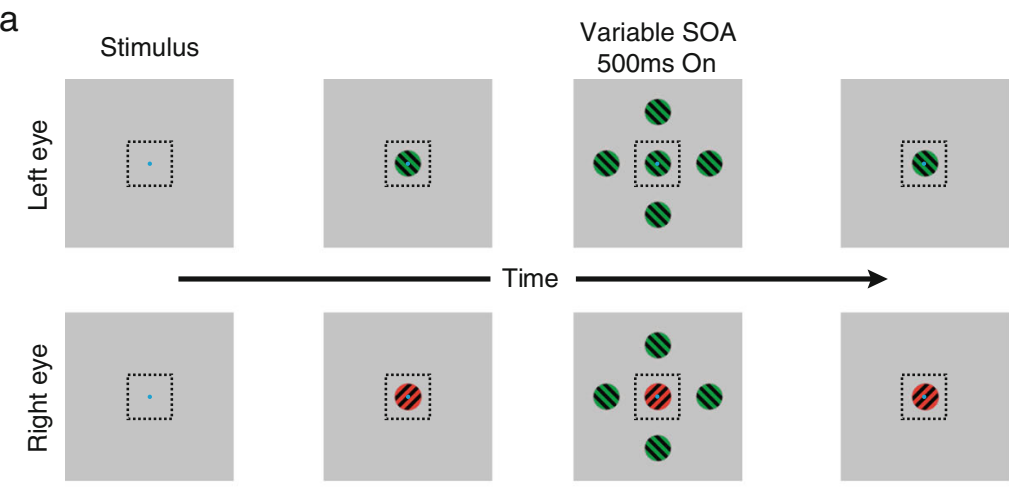

b

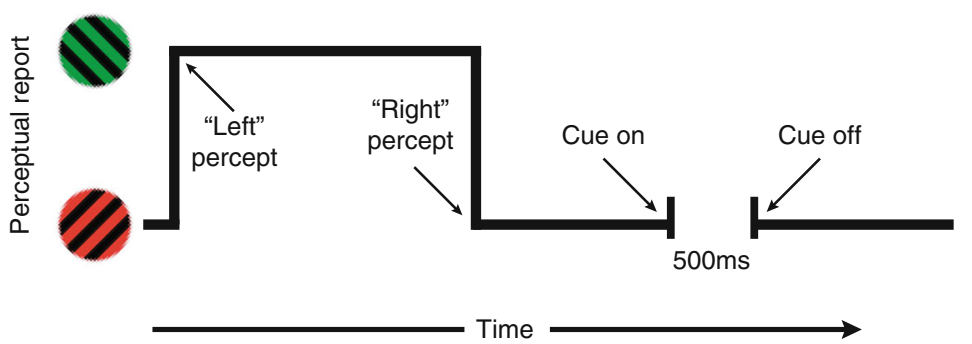

C

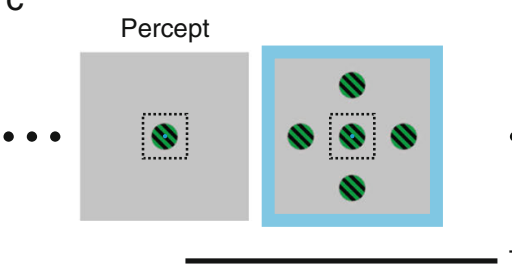

Congruent cue

Fig. 1 Method for Experiment 1: (a) Observers viewed two rival stimuli presented one to each eye. Transient cues were occasionally presented to both eyes. (b) Observers' task was to report the currently dominant percept and ignore surrounding cues when present. Cues were

at the University of Rochester used the following screen settings: $1024 \times 640$ resolution, $120-\mathrm{Hz}$ frame rate, viewing distance $78 \mathrm{~cm}$. For the experiment conducted at Vanderbilt University: $1024 \times 768$ resolution, $100-\mathrm{Hz}$ frame rate, viewing distance $92 \mathrm{~cm}$. Observers viewed stimuli through a mirror stereoscope attached to a chin rest. Prior to the beginning of each session, observers viewed a fusible stimulus and adjusted the alignment of the stereoscope for proper viewing.

\section{Experiment 1}

\section{Stimuli}

Rival stimuli (Fig. 1) were square wave gratings of differing colors (red and green) and orthogonal orientations $( \pm 45)$. The stimulus configuration (i.e., which stimulus was in which eye) d

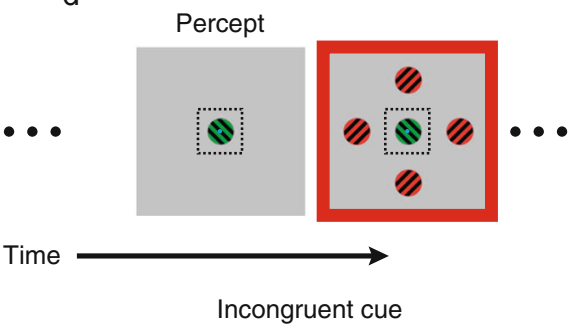

presented for $500 \mathrm{~ms}$ or until the observer indicated a change in percept. The cues could be either congruent (c) or incongruent (d) with the currently reported percept. The colored borders are displayed solely to facilitate matching these conditions to data in Figs. 2 and 3

was determined randomly for each observer but remained constant throughout the experiment. Rival gratings were always surrounded by a square fuser of alternating white and black squares. For all observers, the green grating was fixed at $50 \%$ contrast, while the red grating was set to a perceptually equivalent contrast level (38-100\%, mean $75 \%$, see Procedure). The rival gratings subtended $1^{\circ}$ of visual angle (diameter) and had a spatial frequency of 3.75 cycles ${ }^{\circ}$.

Periodically throughout the experiment, four identical gratings (either red or green, as described above) were presented binocularly in the periphery. These task-irrelevant exogenous cues were centered $2^{\circ}$ from the center of the rival gratings (Fig. 1a), appearing for $500 \mathrm{~ms}$ and then disappearing. The onset time of these cues was variable (see Procedure). 
a

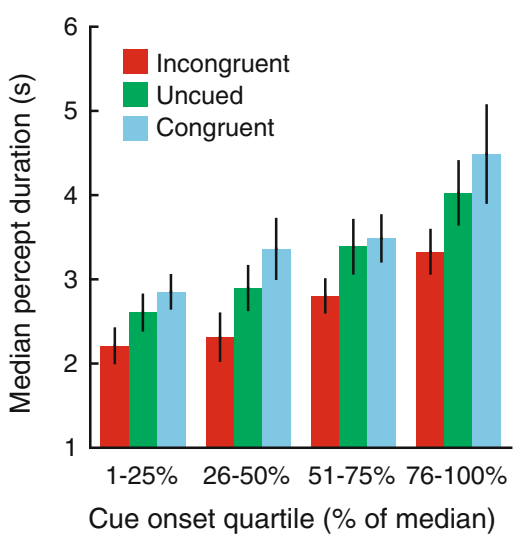

Fig. 2 Effect of transient cues on percept durations: (a) Median percept durations for cued and uncued percepts, grouped by cue onset quartile. For uncued percepts, quartile medians represent the average of expected durations as calculated for each individual cue onset time in the quartile (see Method). (b) Median durations for percepts when no cue was presented (x-axis) vs. those for which a cue did appear (y-axis). Values are calculated by averaging across four quartiles, to account for potential differences across observers and cue conditions in cue onset times. The

\section{Procedure}

Experiment 1 consisted of two 1-hour sessions, each made up of three parts. Observers were instructed to press and hold keys to indicate their dominant percept (right or left tilt), and to release both keys to indicate a mixture of the two images. b

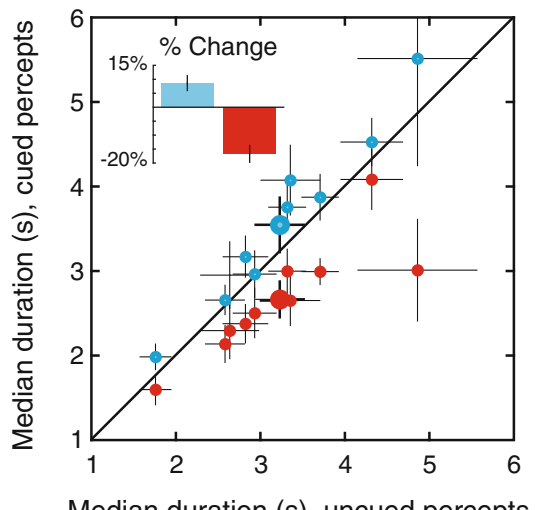

two cue types had highly differentiable effects, with all individual observers showing longer median durations for congruent than incongruent cues. The inset shows the median percent change from expected duration (see Method) for congruent and incongruent cues. Congruent cues led to a $9 \%$ increase in percept duration, while incongruent cues led to a $17 \%$ decrease. Small dots indicate individual medians; large dots are group means; lines indicate SEM

Importantly, observers were instructed to ignore items flashed in the periphery (during part three) and to focus only on the central rival stimuli.

Part 1: The first part of each session was intended to factor out individual differences in eye dominance, and

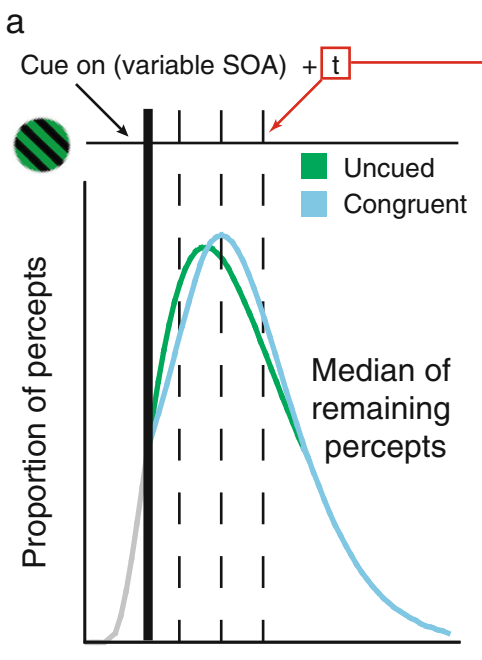

Time b
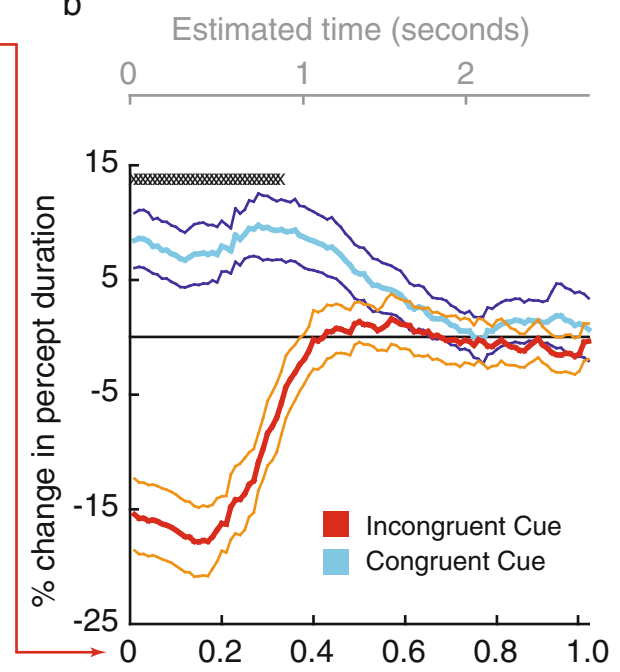

Time, proportion of median duration

indicated a significantly different effect size for the two cue types, $\mathrm{p}<0.01)$. In other words, percepts that were close to ending were significantly modulated by transient cues, while those that would have lasted longer anyway did not differ from expected duration. Group median duration was $2.74 \mathrm{~s}$; significant difference between cue types persists within $0.91 \mathrm{~s}$ ( 0.33 of median duration) of cue onset. Note that the estimate of median duration in Fig. $2 \mathrm{~b}$ is longer ( $3.23 \mathrm{~s}$ ), as averaging across quartiles equally weights short and long percepts even though short percepts are more likely. Thin lines indicate SEM 
to equalize the apparent contrast of the rival stimuli. Observers completed between seven and ten 30-s trials of binocular rivalry tracking. The green grating was fixed at $50 \%$ contrast, while the contrast of the red grating varied across trials. By measuring the proportion predominance of the red grating at various contrast levels, we generated a psychometric function. This was fit with a Weibull function, and the point of subjective equality (PSE) for each observer was then used as the red grating contrast in subsequent parts of the experiment.

Part 2: Using the contrast values obtained in part one, all observers next completed three 2-min trials of rivalry tracking. As in part 1, the stimuli were the rival gratings presented within the fusers. Each observer's median percept duration during this part was used to determine cue onset times in part 3 .

Part 3: The final part of each session consisted of 152 min trials of rivalry tracking. Observers were again instructed to report their percept (right, left, or mixture) while viewing the centrally presented red and green rival gratings. However, during these trials, task-irrelevant exogenous cues were occasionally presented (Fig. 1). These were either four identical gratings matched to the currently reported percept ("congruent" cue, Fig. 1c), or four identical gratings matched to the currently suppressed grating ("incongruent" cue, Fig. 1d). When presented, the gratings were displayed for $500 \mathrm{~ms}$ (Fig. 1b), or until the key press changed (i.e., in the event that the dominant percept changed within $500 \mathrm{~ms}$ ). Cues were never presented during reports of mixed percepts.

Onset times and congruency of the cues were determined randomly prior to each trial, with the constraint that the dominant percept must change either two or three times (both equally likely) between cue onsets. This ensured that (a) cues did not display during consecutive percepts in case influences carried over to subsequent percepts (there was no such effect, see Results) and (b) cues were not always displayed during the same percept.

To determine the timing of the cue onsets, we used the median percept duration established in part two of each session. Possible cue onset times ranged between $1 \%$ and $100 \%$ of the median percept duration, in steps of $1 \%$ (a total of 100 possible onset times for each observer). During session 1, cue onset times were randomly chosen from a uniform distribution of these possible onset times. In the second session, onset times were biased towards later durations - there was a onethird chance of getting an onset between $1 \%$ and $50 \%$ of the median percept duration, and a two-thirds chance of getting an onset between $51 \%$ and $100 \%$ of the median percept duration. This change was intended to counter the increased likelihood of a percept ending before the cue for later cue onsets. To test the effectiveness of this strategy we separated the 100 possible cue onset times into 20 bins consisting of five onset times each. We found that 19/20 bins contained between $4 \%$ and $6.5 \%$ of onset times, meaning cue onset times were relatively uniformly distributed in this experiment. A comparison of quartile results from the two sessions (same as Fig. 2a, but separated by session) indicated no main effect of session $\left(\mathrm{F}_{1,9}=0.04, \mathrm{p}=0.85\right)$ and no interaction $\left(\mathrm{F}_{2,18}=0.96, \mathrm{p}=0.40\right)$, so these data were combined for all further analyses.

\section{Data analysis}

The goal of our analysis was to determine whether presenting congruent or incongruent cues had an influence on percept duration. Because cues could appear with variable SOAs relative to the start of particular percepts, some preprocessing was required. For analyses in Fig. 2, we assumed a motor reaction time (RT) of $350 \mathrm{~ms}$ - any percept ending within $350 \mathrm{~ms}$ after the cue onset was considered to be uncued. The assumption is that any response within $350 \mathrm{~ms}$ of cue onset time was in response to a perceptual change that occurred before the cue onset. For example, for cues presented $800 \mathrm{~ms}$ after a percept's onset, any percept lasting at least $1150 \mathrm{~ms}(800 \mathrm{~ms}+350 \mathrm{~ms}$ RT $)$ was considered a cued percept, while any percepts ending earlier than $1150 \mathrm{~ms}$ were considered uncued. To ensure that our results were not affected by the chosen $350 \mathrm{~ms}$ RT cut-off, we calculated results for a range of possible reaction times and found that the results were unchanged for RTs between 0 and $500 \mathrm{~ms}$.

To quantify how congruent and incongruent cues influenced percept durations, we calculated the percentage change from expectation (Fig. 2 inset, Figs. 3 and 4). Crucially, how long a percept is expected to last depends on the time at which the cue appeared (i.e., how long the percept has already lasted). We defined "expected duration" as the median of all uncued percepts that lasted at least as long as that particular cue onset time (Fig. 3a). This resulted in an increasing expected duration as cues appeared later in the percept. As an analogy, consider the task of computing life expectancy for people of various ages. A person who has already lived 90 years will be expected to live to an older age than a 5-year-old, since we already know that all 90 -year-olds will live at least 90 years.

To determine whether the effects of transient feature-based cues are restricted to a specific time period near the end of individual dominance durations (Fig. 3), we calculated percent change in percept duration while iteratively removing the percepts ending closest in time to the cue onset. Specifically, we first included all cued percepts lasting beyond the cue (leftmost point in Fig. 3b), and then progressively excluded percepts ending after the cue onset in steps of $1 \%$ of each observer's median pretest percept duration. At each step, the cut off used for cued durations was also used to compute a new expected duration (median of uncued percepts lasting at least as long as that cutoff, Fig. 3a). Effect size in 


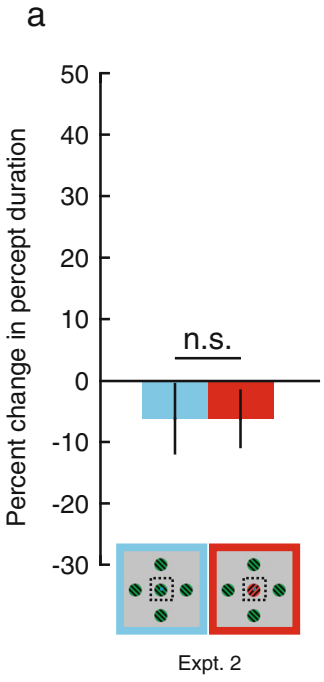

b

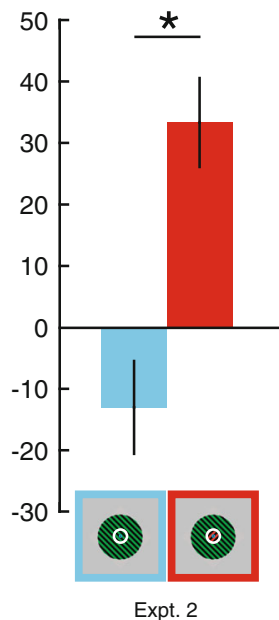

C

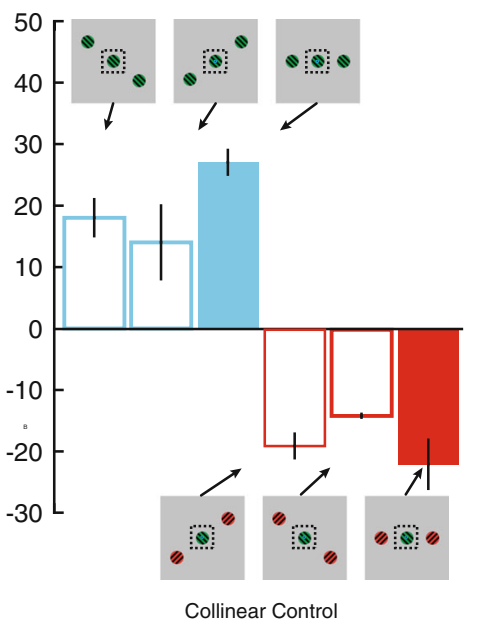

Fig. 4 Results from Experiment 2: We compared spatial interactions of context during binocular rivalry using two types of continuously presented surround stimuli: four surrounding gratings (as in Expt. 1 but continuously presented), (a), and surrounding central rival gratings with an annulus (b). Results showed that without the temporal transience, surrounding rivalry with four gratings had no influence on percept

Fig. 3 indicates the percent change from this iteratively updated expected duration. As the number of data points decreases as we consider progressively longer dominance durations, we restricted this analysis to time bins where we had a sufficient number of samples (generally at least 20 samples per observer). On average, the rightmost bin in Fig. $3 \mathrm{~b}$ contained 56 (congruent), 34 (incongruent) and 147 (uncued) observations per observer. Notably, for the first time point where we found no significant difference between cue types, there are still an average of 136, 109, and 453 observations (respectively) per observer in the analysis.

\section{Experiment 2}

\section{Apparatus and stimuli}

The experimental apparatus was identical to that reported for Experiment 1. Observers viewed two types of stimuli. One set of stimuli matched those reported in Experiment 1 (Fig. 4a) except that the task-irrelevant surround was continuously presented for the entirety of each two-minute trial (i.e., no temporal transients). The other set of stimuli, modeled after (Paffen, Tadin, te Pas, Blake, \& Verstraten, 2006), consisted of the same $1^{\circ}$ diameter central rival stimuli as Experiment 1 , surrounded by a thin white annulus fuser ( 6 arcmin wide). For "surround on" trials, these were continuously surrounded by large fusible annuli oriented at $\pm 45^{\circ}$, and matched in color to the corresponding central rival stimulus. These annuli were matched to the central gratings in spatial frequency (3.75 cycles ${ }^{\circ}$ ) and contrast, and extended $2.5^{\circ}$ in radius from the center of the central grating (see Fig. $4 b$ ). durations (a). However, the same experiment using an attached surrounding annulus showed a significant effect of context on percept durations, in the opposite direction of that found in Experiment 1 (b). (c) Results from the collinear control experiment indicated that altering the spatial arrangement of cues around the central rival stimuli did not impact effect size. ${ }^{*}=$ $\mathrm{p}<0.01$; error bars indicate SEM

\section{Procedure}

Experiment 2 consisted of a single session with two parts. As in Experiment 1, observers first completed a brief calibration test (part 1) to ensure that the two rival gratings were presented at perceptually equivalent contrasts (see Experiment 1 Method).

Part 2: Observers completed nine 2-min trials of rivalry for each surround type (four gratings or annuli). Trials were blocked by surround type, with the order randomly determined for each observer. For both parts of this experiment, peripheral items (when presented) remained constant throughout the 2-min trial. This meant that the same surround, presented for the entirety of a trial, was sometimes congruent and sometimes incongruent with the perceptually dominant grating.

Trial types were identical for both surround types. Three trials were "no surround" trials in which only the central rival gratings were presented with the corresponding fusers; three were "left surround" trials in which the fusible surrounds were tilted at $-45^{\circ}$ (and matched in color to the left tilted central grating); three blocks were "right surround" trials in which the fusible surround were tilted at $+45^{\circ}$ (and matched in color to the right tilted central grating). Trials were presented in a random order within each block. For all parts and for all trials, observers were again instructed only to report the perceptual alternations of the central rival stimuli, and to ignore the surround. 


\section{Results}

\section{Experiment 1}

Before turning to the temporal dynamics that are the primary focus of this study, we first determined whether the taskirrelevant exogenous cues in fact influenced perception during rivalry. Because cue SOAs were randomly distributed, data for each observer were first grouped by quartiles of cue onset times (Fig. 2a). In addition to an anticipated main effect of quartile (see Method), a two-factor ANOVA indicated a significant effect of cue type $\left(\mathrm{F}_{2,18}=17, \mathrm{p}<0.0001\right)$ such that incongruent cues shortened and congruent cues lengthened dominance durations during which cues were presented. There was no interaction between quartile and percept duration $\left(\mathrm{F}_{6,54}=1.68, \mathrm{p}=0.14\right)$. However, as shown by the temporal analyses detailed below, this does not preclude temporal specificity of cue effects.

To focus on the observed main effect of cue type, we directly compared percept durations when cues were presented to those when no cue was presented, collapsing across all cue onset quartiles. Results again indicated a significant effect of cue type $\left(\mathrm{F}_{2,18}=17, \mathrm{p}<0.001\right)$ that was driven by significantly longer percepts when congruent cues were presented $\left(t_{9}=4.4\right.$, $\mathrm{p}<0.01)$ and significantly shorter percepts when incongruent cues were presented $\left(\mathrm{t}_{9}=-3.7, \mathrm{p}<0.01\right)$, as compared to uncued percepts (Fig. 2b). Direct comparison of percent change from expected duration (Fig. 2b inset; see Method) again revealed a significant difference between congruent and incongruent cues $\left(t_{9}=9.3, p<0.0001\right)$. The direction of this difference was the same for all ten observers individually (Fig. 2b). On average (Fig. 2b inset), we found a moderate but significant effect of congruent cues ( $9 \%$ increase in percept duration) and a larger effect of incongruent cues (17\% decrease in percept duration).

To establish whether or not it matters "when" during a given percept the cue is presented, we investigated the temporal dependence of the observed cueing effect. The stochastic nature of binocular rivalry makes such an analysis difficult because we do not know in advance when a given dominance duration will end. To circumvent this problem we conducted the following analysis (Fig. 3a). If cues act effectively only on percepts that are near their end (as hypothesized), then the effect of the cue should be seen only close in time to the cue onset. In other words, the biggest effects will be seen for cued percepts relative to uncued percepts that ended shortly after the cue onset time, as those were the percepts that were extended or shortened (note that this rationale holds because the effects of the cues are smaller than the typical dominance durations, as shown in Fig. 2).

Beginning by including all percepts ending after the cue onset (thick line in Fig. 3a; leftmost point in Fig. 3b), we progressively excluded percepts ending after the cue onset in steps of $1 \%$ of each observer's median percept duration. Thus, the rightmost point (1.0 median on $\mathrm{x}$-axis, Fig. 3b) shows the percentage change in percept duration (cued duration/expected duration * $100-100$ ) for all points that lasted at least a median dominance duration beyond the onset of the cue - any percepts that ended within a median dominance duration following the cue onset would now be removed. We also performed an equivalent analysis on raw dominance durations, and the results were essentially identical.

This analysis shows that the differential effects of congruent and incongruent cues (see Fig. 2) are present only if those percepts that end close in time to the cue onset are included (within 0.33 median dominance durations, or $\sim 0.91 \mathrm{~s}$, of cue). In other words, those percepts that lasted long beyond the cue were not significantly influenced by the cues. In sum, the main selective effects of the cues are largely carried by dominance durations that end within about $1 \mathrm{~s}$ of the cue onset, indicating that the cues have an effect only if presented near the end of individual dominance durations.

While these results support our hypothesis that binocular rivalry is most susceptible to selective modulation near the end of individual dominance periods, another explanation is that the temporally focused effect of the cues is simply a consequence of the brief cue duration. Indeed, sustained effects of surround context have been reported before (Paffen, Tadin, et al., 2006; Sobel \& Blake, 2002). To address this alternative explanation, we conducted a control experiment $(\mathrm{N}=4$, also participated in main experiment) in which the cues flashed on as before, but remained on the screen until the reported percept changed. Thus, we retained the salient cue onset, but the cues remained throughout the cued dominance duration, maximizing the opportunity to reveal any sustained effects. Nevertheless, we found no significant differences in the results. A two-factor ANOVA (context type, experiment) comparing percent change from expectation again showed a main effect of cue type $\left(\mathrm{F}_{1,3}=24.8, \mathrm{p}=0.02\right)$, but indicated that effects of cues on percept durations did not differ between experiments $\left(\mathrm{F}_{1,3}=3.8, \mathrm{p}=0.15\right)$ and that there was no interaction between cue type and experiment $\left(\mathrm{F}_{1,3}=0.6, \mathrm{p}=0.49\right)$. To further test for the transient nature of the observed effects, we investigated whether cue effects carried over to subsequent percept durations. Specifically, using data from Experiment 1, we tested whether the subsequent percept was systematically influenced by the cue that had been presented during the previous percept. We found that uncued percepts immediately preceded by congruent cues did not differ in duration from those immediately preceded by incongruent cues $\left(t_{9}=0.8\right.$, $\mathrm{p}=0.47$ ). Taken together, these findings demonstrate that the effects of congruent and incongruent cues are transient, affecting only percept durations that would have ended shortly after the cue onset. These results are consistent with the engagement of exogenous, feature-based attention towards rival 
features that match the presented cue, even if the rival feature is suppressed from awareness (cf. Koch \& Tsuchiya, 2007; Lamme, 2003). However, these results are also consistent with transiently driven spatial interactions between the surrounding cues and central rivalry. We test this alternative hypothesis in Experiment 2.

\section{Experiment 2}

The direction of the observed cue effects in Experiment 1 differs from previous reports where congruent context shortened and incongruent context lengthened dominance durations (Fukuda \& Blake, 1992; Paffen, Tadin, et al., 2006). Those results, taken to indicate sustained suppressive effects of context rather than transient exogenous attention, differed in two important ways: context was presented continuously throughout rivalry, and was spatially adjacent to the rival stimuli. To address these differences, we first continuously presented the peripheral context from Experiment 1 during each 120 -s trial of binocular rivalry (Fig. 4a). Under these conditions, there was no differential effect of the cues $\left(t_{6}=0, p=1\right)$. In contrast, when the context was directly adjacent to and fully surrounded the rival stimuli (Fig. 4b), we found a strong effect of context type $\left(\mathrm{t}_{6}=-3.8, \mathrm{p}<0.01\right.$; Fig. $\left.4 \mathrm{~b}\right)$, replicating contextual effects reported previously (Fukuda \& Blake, 1992; Paffen, Tadin, et al., 2006). The direction of this effect, however, was opposite to what we found with the transient cues (Fig. 2b inset and Fig. 4b; significant interaction between experiment and cue type, $\left.\mathrm{F}_{1,15}=53, \mathrm{p}<0.0001\right)$. These findings provide further evidence that the transient nature of cues used in Experiment 1 drives the observed effects, dissociating those effects from previous reports of contextual modulation during binocular rivalry.

To further test for the role of spatial interactions, we explored the effects of cue-target collinearity. Collinearity plays a strong role in spatial interactions between stimuli (Kapadia, Ito, Gilbert, \& Westheimer, 1995) and is also known to impact rivalry dynamics (Alais, Lorenceau, Arrighi, \& Cass, 2006). Thus, if such spatial interactions underlie our results, stimulus manipulations that alter feature collinearity should also affect the (in)congruency effects observed in Experiment 1. To test this hypothesis, we conducted a separate control experiment $(\mathrm{N}=2)$, where two transiently presented congruent or incongruent cues (as in Experiment 1) were positioned collinearly, orthogonally, or placed on the left and right (as in Experiments 1 and 2) relative to the rival stimuli (Fig. 4c). Collinearity was determined with respect to the rival grating matching the features of the cue. We again found a significant effect of cue type $\left(F_{1,1}=478, p=0.03\right)$, but no effect of collinearity $\left(F_{2,2}=0.9\right.$, $\mathrm{p}=0.52)$ and no interaction $\left(\mathrm{F}_{2,2}=2.3, \mathrm{p}=0.3\right)$. In sum, control conditions in Experiment 2 indicate that the main effects observed in Experiment 1 are not caused by spatial interactions between cues and rival targets (see Discussion for more details).

\section{Discussion}

Our results demonstrate that task-irrelevant exogenous cues can lead to feature-specific modulations of percept durations during binocular rivalry. Presenting a cue with features matched to the currently dominant percept prolongs the dominance of that percept, while presenting a cue matched to the suppressed stimulus results in shorter percept durations. As detailed below, these effects are likely driven by exogenous feature-based attention that strengthened the rival target matching the cue features. Contextual modulations per se cannot explain these effects, as they disappeared when the temporal transience was eliminated (Experiment 2, Fig. 4a), and did not increase in magnitude when context was presented for a longer total duration of time (Experiment 1, Results; Fig. 4a). Moreover, the effects observed in Experiment 1 were opposite those typically found in experiments of contextual spatial interactions during binocular rivalry (Fig. 4b; Fukuda \& Blake, 1992; Paffen, Tadin, et al., 2006; Paffen, Van Der Smagt, te Pas, \& Verstraten, 2005), and opposite those seen in a similar spatial display when flanking stimuli appear gradually (Kim, Shin, \& Chong, 2013). These results, coupled with the absence of collinearity effects (Fig. 4c), suggest that our results are not driven by spatial interaction effects. We speculate that the presence of a high contrast fusion stimulus between the rival targets and cues (Fig. 1) attenuated spatial interaction effects by facilitating perceptual segregation of cues and targets (cf. Tadin, Paffen, Blake, \& Lappin, 2008).

The key result of this study is that cue effectiveness was largely explained by when the cues appeared. Results showed that cues only influenced the durations of percepts that were within about a third of an observer's median percept duration ( $\sim 1 \mathrm{~s}$ ) of ending (Fig. 3b) had the cue not been displayed. This finding supports the notion that even as one image remains perceptually dominant, the relative strength of dominance and suppression between rival stimuli in fact converges (Alais et al., 2010). In turn, this suggests that rivalry is less clearly resolved during these late portions of a perceptual dominance epoch, resulting in a greater degree of selective control (Dieter \& Tadin, 2011).

Our finding that selective influences during binocular rivalry are limited to periods when rival competition is least resolved may provide a link between binocular rivalry and other paradigms used to study visual attention. Early in a percept, when rivalry conflict is largely resolved in favor of the dominant stimulus (Alais et al., 2010), attention has little ability to perceptually select one of the two inputs (also see McMains \& Kastner, 2011). However, as the percept extends in time, unresolved competition creeps back in (Alais et al., 2010) and 
attentional effects are unlocked. In addition to highlighting the adherence of binocular rivalry to general principles governing visual selective attention (Desimone \& Duncan, 1995), our results also reveal the utility of attentional studies for elucidating mechanisms underlying complex visual processes.

Our hypothesis is based on an extension of the biased competition theory of attention (which typically applies to spatially separate stimuli) to rival stimuli that occupy the same location in visual space (Dieter \& Tadin, 2011). For the early visual areas (i.e., areas with small receptive fields) thought to be critical in the resolution of binocular rivalry (Blake, 1989), biased competition theory predicts the strongest competition for stimuli that are the closest in space (Kastner, De Weerd, Desimone, \& Ungerleider, 1998; Kastner et al., 2001). In our case, this would be stimuli that spatially overlap. Moreover, because our rival stimuli were both spatially separated (by $2^{\circ}$ ) and perceptually segregated (via the fusion stimulus) from cue stimuli, it is fair to assume that the competition between cues and rival stimuli is considerably smaller than the competition between rival stimuli.

The temporal specificity of these results is consistent with the idea that our cues promote the deployment of exogenous feature-based attention (cf. Lin, Hubert-Wallander, Murray, \& Boynton, 2011) to the rival stimulus that matches the cue. Indeed, exogenous shifts in spatial attention have previously been shown to cause shorter percept durations (Paffen \& Van der Stigchel, 2010), but our results are the first indication that extended binocular rivalry can be exogenously influenced in a manner that depends on the features of the exogenous cue. Such effects have been previously demonstrated for initial rivalry (Chong \& Blake, 2006), known to be more susceptible to attentional modulation than later phases of binocular rivalry (Dieter \& Tadin, 2011). This suggests that the competitive interactions during ongoing binocular rivalry may return to a state similar to initial rivalry near the end of a given percept's dominance phase (Alais et al., 2010).

Results revealed stronger effects of incongruent than congruent cues (Figs. 2 and 3). We speculate that this effect size asymmetry reflects additional effects of non-selective attentional influences over rivalry dynamics. Increased attention or shifts of attention should lead to a general reduction in percept durations (Paffen, Alais, \& Verstraten, 2006; Paffen \& Van der Stigchel, 2010). The same would be expected if cue presentation caused an increase in eye movements (Peckham, 1936; van Dam \& van Ee, 2006). Combining these non-selective speeding effects with cue-specific effects of feature-based attention would result in an overall speeding of rivalry, and, thus, stronger effects for incongruent cues (which cause additional speeding). Notably, for two experienced psychophysical observers in the collinearity control experiment, effect sizes were similar for congruent and incongruent cues (Fig. 4c). For eye movements to fully account for the effects we observe, however, the eyes would need to move in a manner that changes the retinal image in only one of the two eyes; stimulus dominance can be prolonged by retinal image shifts that are restricted to the dominant eye (and vice versa; van Dam \& van Ee, 2006). For our grating stimuli, such selective image shifts could result from cue-induced eye movements that are parallel to one of the two rival gratings. However, such a pattern of eye movements is unlikely given the spatial arrangement of cues in Experiment 1 because eye movements toward any cue location would not be parallel to either rival pattern. More importantly, we found that our main results were not affected by changes the spatial arrangement of the cues (Fig. 4c), further arguing against an eye movement based account.

How might exogenous feature-based attention modulate percept durations in our paradigm? Increases in the effective contrast of rivalry stimuli, like physical contrast increases (Levelt, 1965), can influence rivalry dynamics (Dieter \& Tadin, 2011; Paffen \& Alais, 2011) by influencing the speed of rivalry alternations (Paffen \& Van der Stigchel, 2010). In order to selectively influence binocular rivalry in the way we demonstrate, however, feature-based attention would need to increase the contrast of only the rival stimulus with matching features. Thus, the increase in percept duration that we observe with congruent cues is consistent with a boost in effective stimulus contrast only to the currently dominant image (Chong et al., 2005; Mueller \& Blake, 1989).

Likewise, an increase in effective contrast for the suppressed image when an incongruent cue is presented would also cause it to break through suppression more quickly (Levelt, 1965), thereby more rapidly ending the currently dominant percept and leading to the results we observe. For attention to drive such an effect, it would require attention to features that are perceptually suppressed. There is indeed evidence that behaviorally relevant stimuli, which may attract attention more than neutral stimuli, can break through perceptual suppression faster (Alpers \& Pauli, 2006; Anderson, Siegel, Bliss-Moreau, \& Barrett, 2011; Bannerman, Milders, de Gelder, \& Sahraie, 2008; Yang, Zald, \& Blake, 2007). Our results indicate that exogenous attentional cues may drive attention to suppressed features, providing further evidence for the dissociation of attention and consciousness (Koch \& Tsuchiya, 2007; Lamme, 2003).

This finding also sheds light on an intriguing pattern of attentional results that is typically found in binocular rivalry and attention experiments. Simply trying to "hold" one of two percepts dominant while viewing binocular rivalry is ineffective (Blake, 1988; Meng \& Tong, 2004), but observers can selectively influence rivalry dynamics with attention when performing a demanding perceptual task (Chong et al., 2005; Hancock \& Andrews, 2007; Helmholtz, 1925). The findings of Chong et al. suggest that the direction of attention towards the features of one of the two rival stimuli is crucial. Our results extend this finding by showing that the time at which 
feature-based attention is directed towards a rival stimulus is also essential. With this in mind, observers" failure to "hold" a stimulus during binocular rivalry (Meng \& Tong, 2004) may reflect difficulties in sustaining attention for the duration of a psychophysical experiment without a demanding task (Lavie, 2005). If observers are asked to perform a demanding task during binocular rivalry (e.g. Chong et al., 2005), this likely ensures that observers direct feature-based attention towards the relevant rival stimulus at the critical time (i.e., the final 1-s of its dominance).

To summarize, we show that transiently presented featurebased cues can induce selective modulations of binocular rivalry dynamics. They seem to do so by strengthening the rival stimulus with features matching those of the presented cue, likely through the mechanism of feature-based attention. Crucially, these modulations depend on precise timing of the transient cues, as cues presented more than $1 \mathrm{~s}$ before the end of a given percept were shown to have no influence. These findings provide further evidence that the strength of dominance and suppression in binocular rivalry are not fixed, but instead converge over the course of a percept's dominance phase (Alais et al., 2010). This leads to increased competition between stimuli near the end of the percept, which is more susceptible to attentional influence over perception (Dieter \& Tadin, 2011).

Acknowledgments The authors thank Randolph Blake for helpful comments on the manuscript. This work was supported by NIH NEI T32 EY 007135-19 (to VVRC) and T32 EY 7125-22 (to CVS).

KD \& DT developed the study concept. All authors contributed to the study design. Testing and data collection were performed by KD. KD performed the data analysis and interpretation under the supervision of DT. KD drafted the manuscript, and MM and DT provided critical revisions. All authors approved the final version of the manuscript for submission.

\section{References}

Alais, D., Cass, J., O'shea, R. P., \& Blake, R. (2010). Visual sensitivity underlying changes in visual consciousness. Current Biology, 20(15), 1362-1367.

Alais, D., Lorenceau, J., Arrighi, R., \& Cass, J. (2006). Contour interactions between pairs of Gabors engaged in binocular rivalry reveal a map of the association field. Vision Research, 46(8-9), 1473-1487.

Alpers, G., \& Pauli, P. (2006). Emotional pictures predominate in binocular rivalry. Cognition \& Emotion, 20(5), 596-607.

Anderson, E., Siegel, E. H., Bliss-Moreau, E., \& Barrett, L. F. (2011). The visual impact of gossip. Science, 332(6036), 1446-1448.

Bannerman, R. L., Milders, M., de Gelder, B., \& Sahraie, A. (2008). Influence of emotional facial expressions on binocular rivalry. Ophthalmic and Physiological Optics, 28(4), 317-326.

Beck, D. M., \& Kastner, S. (2009). Top-down and bottom-up mechanisms in biasing competition in the human brain. Vision Research, 49(10), 1154-1165.

Blake, R. (1988). Dichoptic reading: The role of meaning in binocular rivalry. Perception \& Psychophysics, 44(2), 133-141.
Blake, R. (1989). A neural theory of binocular rivalry. Psychological Review, 96(1), 145.

Brainard, D. H. (1997). The Psychophysics Toolbox. Spatial Vision, 10(4), 433-436.

Chong, S. C., \& Blake, R. (2006). Exogenous attention and endogenous attention influence initial dominance in binocular rivalry. Vision Research, 46(11), 1794-1803.

Chong, S. C., Tadin, D., \& Blake, R. (2005). Endogenous attention prolongs dominance durations in binocular rivalry. Journal of Vision, 5(11), 1004-1012.

Desimone, R., \& Duncan, J. (1995). Neural mechanisms of selective visual attention. Annual Review of Neuroscience, 18(1), 193-222.

Dieter, K. C., \& Tadin, D. (2011). Understanding attentional modulation of binocular rivalry: a framework based on biased competition. Frontiers in Human Neuroscience, 5, 1-12.

Fukuda, H., \& Blake, R. (1992). Spatial interactions in binocular rivalry. Journal of Experimental Psychology: Human Perception and Performance, 18(2), 362-370.

Hancock, S., \& Andrews, T. J. (2007). The role of voluntary and involuntary attention in selecting perceptual dominance during binocular rivalry. Perception-London, 36(2), 288-298.

Helmholtz, H. v. (1925). Treatise on Physiological Optics, Vol. 3 (J. P. C. Southall, Trans.). New York: Dover.

Hol, K., Koene, A., \& van Ee, R. (2003). Attention-biased multi-stable surface perception in three-dimensional structure-from-motion. Journal of Vision, 3(7), 486-498.

Kapadia, M. K., Ito, M., Gilbert, C. D., \& Westheimer, G. (1995). Improvement in visual sensitivity by changes in local context: parallel studies in human observers and in V1 of alert monkeys. Neuron, 15(4), 843-856.

Kastner, S., De Weerd, P., Desimone, R., \& Ungerleider, L. G. (1998). Mechanisms of directed attention in the human extrastriate cortex as revealed by functional MRI. Science, 282(5386), 108-111.

Kastner, S., De Weerd, P., Pinsk, M. A., Elizondo, M. I., Desimone, R., \& Ungerleider, L. G. (2001). Modulation of sensory suppression: implications for receptive field sizes in the human visual cortex. Journal of Neurophysiology, 86(3), 1398-1411.

Kim, S., Shin, E., \& Chong, S. C. (2013). When crowding meets binocular rivalry: Challenges for object perception. Vision Research, 76, 134-143.

Koch, C., \& Tsuchiya, N. (2007). Attention and consciousness: two distinct brain processes. Trends in Cognitive Sciences, 11(1), 16-22.

Lamme, V. A. (2003). Why visual attention and awareness are different. Trends in Cognitive Sciences, 7(1), 12-18.

Lavie, N. (2005). Distracted and confused? Selective attention under load. Trends in Cognitive Sciences, 9(2), 75-82.

Levelt, W. (1965). On Binocular Rivalry. Paris: Mouton

Lin, J. Y., Hubert-Wallander, B., Murray, S. O., \& Boynton, G. M. (2011). Rapid and reflexive feature-based attention. Journal of Vision, 11(12), 1-9.

McMains, S., \& Kastner, S. (2011). Interactions of top-down and bottomup mechanisms in human visual cortex. The Journal of Neuroscience, 31(2), 587-597.

Meng, M., \& Tong, F. (2004). Can attention selectively bias bistable perception? Differences between binocular rivalry and ambiguous figures. Journal of Vision, 4(7), 539-551.

Mitchell, J. F., Stoner, G. R., \& Reynolds, J. H. (2004). Object-based attention determines dominance in binocular rivalry. Nature, 429(6990), 410-413.

Mueller, T. J., \& Blake, R. (1989). A fresh look at the temporal dynamics of binocular rivalry. Biological Cybernetics, 61(3), 223-232.

Necker, L. A. (1832). Observations on some remarkable optical phaenomena seen in Switzerland; and on an optical phaenomenon which occus on viewing a figure of a crystal or geometrical solid. Philosophical Magazine Series, 3, 1(5), 329-337. 
Ooi, T. L., \& He, Z. J. (1999). Binocular rivalry and visual awareness: The role of attention. Perception-London, 28(5), 551-574.

Paffen, C. L., \& Alais, D. (2011). Attentional modulation of binocular rivalry. Frontiers in Human Neuroscience, 5, 1-10.

Paffen, C. L., Alais, D., \& Verstraten, F. A. (2006). Attention speeds binocular rivalry. Psychological Science, 17(9), 752-756.

Paffen, C. L., Tadin, D., te Pas, S. F., Blake, R., \& Verstraten, F. A. (2006). Adaptive center-surround interactions in human vision revealed during binocular rivalry. Vision Research, 46(5), 599-604.

Paffen, C. L., Van Der Smagt, M. J., te Pas, S. F., \& Verstraten, F. A. (2005). Center-surround inhibition and facilitation as a function of size and contrast at multiple levels of visual motion processing. Journal of Vision, 5(6), 571-578.

Paffen, C. L., \& Van der Stigchel, S. (2010). Shifting spatial attention makes you flip: exogenous visual attention triggers perceptual alternations during binocular rivalry. Perception \&amp; Psychophysics, 72(5), 1237-1243.

Peckham, R. H. (1936). Eye movements during "retinal rivalry". American Journal of Psychology, 48, 43-63.

Pelli, D. G. (1997). The VideoToolbox software for visual psychophysics: transforming numbers into movies. Spatial Vision, 10(4), 437-442.

Sobel, K. V., \& Blake, R. (2002). How context influences predominance during binocular rivalry. Perception, 31(7), 813-824.
Stuit, S. M., Paffen, C. L., Van Der Smagt, M. J., \& Verstraten, F. A. (2014). Image-based grouping during binocular rivalry is dictated by eye-of-origin. PloS One, 9(7), e95327.

Sundareswara, R., \& Schrater, P. R. (2008). Perceptual multistability predicted by search model for Bayesian decisions. Journal of Vision, $8(5), 1-19$.

Suzuki, S., \& Peterson, M. A. (2000). Multiplicative effects of intention on the perception of bistable apparent motion. Psychological Science, 11(3), 202-209.

Tadin, D., Paffen, C. L., Blake, R., \& Lappin, J. S. (2008). Contextual modulations of center-surround interactions in motion revealed with the motion aftereffect. Journal of Vision, 8(7), 1-11.

Toppino, T. C. (2003). Reversible-figure perception: Mechanisms of intentional control. Perception \&amp; Psychophysics, 65(8), 12851295.

van Dam, L. C., \& van Ee, R. (2006). Retinal image shifts, but not eye movements per se, cause alternations in awareness during binocular rivalry. Journal of Vision, 6(11), 1172-1179.

Wolfe, J. M. (1983). Influence of spatial frequency, luminance, and duration on binocular rivalry and abnormal fusion of briefly presented dichoptic stimuli. Perception, 12(4), 447-456.

Yang, E., Zald, D. H., \& Blake, R. (2007). Fearful expressions gain preferential access to awareness during continuous flash suppression. Emotion, 7(4), 882-886. 